\title{
Composition and Properties of Maple Sap, Concentrate, and Permeate
}

\author{
Abby K. van den Berg1, Timothy D. Perkinss ${ }^{1}$, Mark L. Isselhardt ${ }^{2}$ \\ ${ }^{1}$ Proctor Maple Research Center, The University of Vermont, Underhill, VT, USA \\ ${ }^{2}$ The University of Vermont Extension, Underhill, VT, USA \\ Email: Abby.vandenBerg@uvm.edu
}

How to cite this paper: van den Berg, A.K. Perkins, T.D. and Isselhardt, M.L. (2019) Composition and Properties of Maple Sap, Concentrate, and Permeate. Agricultural Sciences, 10, 32-45.

https://doi.org/10.4236/as.2019.101004

Received: November 25, 2018

Accepted: January 7, 2019

Published: January 10, 2019

Copyright () 2019 by author(s) and Scientific Research Publishing Inc. This work is licensed under the Creative Commons Attribution International License (CC BY 4.0).

http://creativecommons.org/licenses/by/4.0/

\begin{abstract}
Reverse osmosis is used widely in the maple syrup industry to concentrate maple sap and increase the overall efficiency and profitability of syrup production. Sets of samples from maple producers utilizing a range of sap concentration levels were collected and analyzed to provide a portrait of the physicochemical properties and chemical composition of maple sap, concentrate, and permeate across a single production season. The results reinforce that reverse osmosis functions essentially as a concentration process, without significantly altering the fundamental proportions of sap constituents.
\end{abstract}

\section{Keywords}

Maple Syrup, Reverse Osmosis, Membrane Separation, Maple Sap

\section{Introduction}

Reverse osmosis (RO) is a widely-used technology in the maple syrup industry ${ }^{1}$. In general, it is used to increase production efficiency by removing water from maple sap, which has a sugar content of approximately $2 \%$, and reducing the amount of time and fuel required to complete concentration to syrup density (66\% - 68\%) through thermal atmospheric evaporation in maple syrup evaporators [1]. Since its introduction into the industry in the 1970's, there were some concerns expressed that reducing the amount of time sap was processed with heat in evaporators could negatively impact the flavor or other unique properties of maple syrup, as the majority of the reactions responsible for flavor, aroma, and color development in maple syrup occur as a result of thermal processes, primarily carbohydrate decomposition and Maillard reactions [1]. As such, there

${ }^{1}$ The term "reverse osmosis" is used in the maple industry to refer to any type of membrane separation, although nanofiltration is the primary type currently used. 
have been several studies conducted and published on the effects of this technology on syrup composition and flavor which have found no substantive impacts [2]-[8]. Despite this, still somewhat limited in the published literature are basic data on the composition and physicochemical properties of concentrated sap, as well as the sap from which it was derived and the permeate water generated during the process [1] [9] [10]. Thus, the objective of this work was to provide a portrait of the composition and physicochemical properties of concentrated sap, as well as the sap from which it was derived and the permeate generated during concentration.

\section{Materials and Methods}

\subsection{Sample Collection}

Twenty maple producers in Vermont were recruited to participate in the study during the 2009 production season, 5 each that typically concentrated their sap in the following 4 ranges: $8 \%-11 \%, 12 \%-15 \%, 16 \%-20 \%$, and $21 \%-25 \%$ sugar, respectively. The objective was not to provide a representative sample of membrane types, configurations, or RO equipment, but simply a snapshot of the common ranges of concentration that producers were typically using at the time. Prior to the production season, the producers were provided with instructions and a set of glass sample collection bottles. They were instructed to collect samples of raw sap ("sap"), and the concentrated sap ("concentrate") and water ("permeate") produced from that sap at 3 times during the production season, at timepoints representative of the early, middle, and late portions of their production seasons. The duration and timing of the maple production season varies annually and between individual operations due to differences in climate and operational factors. As such, producers were instructed to collect these samples at times representative of the first, middle, and last thirds of the production seasons of their individual operations. Producers were instructed to collect the set of sap, concentrate, and permeate samples simultaneously, and to place them in a freezer immediately. At the end of the production season, all samples were collected from each producer and placed in a freezer at the University of Vermont Proctor Maple Research Center (PMRC) in Underhill, Vermont. Analyses to determine the physiochemical properties and chemical composition of the samples were conducted during the summer of 2009.

Several samples were lost prior to analyses due a variety of factors such as producer freezer malfunction or spoilage prior to transport to PMRC. Thus, there are several producers for which there is not a complete set of data from early, middle, and late timepoints, and all samples from some producers were lost. Ultimately, 34 sets of sap, concentrate and permeate samples from 14 producers were analyzed and included in the analyses.

\subsection{Sample Analyses}

\subsubsection{Physicochemical Properties}

Electrical conductivity $(\mu \mathrm{S} / \mathrm{cm}, \pm 0.005 \%)$ and $\mathrm{pH}( \pm 0.01)$ of samples were de- 
termined with an Accumet XL60 meter using an Accumet epoxy body electrical conductivity cell and an Accufet XL solid-state pH probe, respectively, both equipped with automatic temperature compensation (Fisher Scientific, Fair Lawn, NY, USA). Sugar content $\left( \pm 0.1^{\circ}\right.$ Brix $)$ of samples was measured with a PA203X handheld digital refractometer (Misco, Cleveland, OH, USA).

\subsubsection{Inorganic Minerals}

The mineral composition was determined for each sap and concentrate sample, and a subset of permeate samples from 3 producers. The concentrations of boron, calcium, copper, iron, magnesium, manganese, phosphorous, potassium, sodium, sulfur and zinc (ppm, $\pm \sim 5 \%-15 \%$ ) were determined by inductively coupled plasma atomic emission spectroscopy (ICP-AES) with a PlasmaSpec 2.5 (Leeman Labs, Hudson, NH, USA). Concentrate samples were digested prior to analysis; $0.5 \mathrm{~g}$ of each sample was digested with $10 \mathrm{~mL}$ concentrated nitric acid for $15 \mathrm{~min}$ at $190^{\circ} \mathrm{C}$ and $2.1 \mathrm{MPa}$ pressure. The detection limits (ppm) for each element in sap and permeate samples were: $\mathrm{K}=1.0 ; \mathrm{Ca}, \mathrm{Mg}, \mathrm{B}, \mathrm{S}=0.05 ; \mathrm{P}, \mathrm{Na}=$ $0.1 ; \mathrm{Cu}, \mathrm{Zn},=0.02 ; \mathrm{Fe}, \mathrm{Mn}=0.01$. Detection limits (ppm) for concentrate samples were: $\mathrm{Ca}, \mathrm{P}, \mathrm{K}, \mathrm{Mg}, \mathrm{Na}, \mathrm{S}=1.0 ; \mathrm{Fe}, \mathrm{Mn}, \mathrm{B}, \mathrm{Cu}, \mathrm{Zn}=0.05$. Minerals for which the concentrations in greater than $20 \%$ of a sample type were below the detection limit were excluded from analyses. All $\mathrm{B}$ values for sap samples, $\mathrm{Na}$ values for concentrate samples, and $\mathrm{P}, \mathrm{Mg}, \mathrm{Fe}, \mathrm{B}, \mathrm{Cu}, \mathrm{Zn}$, and $\mathrm{S}$ values for permeate samples, were below the detection limit of the instrument. For other samples which were below the detection limit, values were set at the detection limit divided by the square root of 2 [11]. The number of samples for each mineral for each sample type calculated in this manner was: permeate $=\mathrm{Ca}(2), \mathrm{Na}(2), \mathrm{Mn}$ (2); sap = Na (3), Fe (2), S (1); concentrate = B (1), S (8).

\subsubsection{Carbohydrates}

The composition of sucrose, glucose, and fructose (total percentage) in each sap and concentrate sample was determined by high-performance liquid chromatography (HPLC) using a 1525 binary pump and 2410 refractive index detector (Waters, Milford, MA, USA). An Aminex HPX-87K column $(300 \times 7.8 \mathrm{~mm}$, Bio-Rad, Hercules, CA, USA) was used at $75^{\circ} \mathrm{C}$ with a mobile phase of $0.2 \mathrm{~mm}$ potassium phosphate at a rate of $0.6 \mathrm{~mL}$ min-1. The carbohydrate composition of the permeate samples from a subset of 3 producers was also determined.

\section{Results and Discussion}

\subsection{Overall Means}

Table 1 presents the overall mean, minimum, and maximum composition and properties for all sap, concentrate, and permeate samples. Average concentration levels of the concentrate samples from the 14 producers ranged from 8.9 to 19.8 ${ }^{\circ} \mathrm{Brix}$, with average $\mathrm{pH}$ values slightly below neutral, 6.96 , and ranging from just below to slightly above neutral (Table 1). Further interpretation of overall mean composition values for the concentrate samples is not possible due to the wide 
Table 1. Overall mean ( \pm standard error of the mean), minimum, and maximum physicochemical properties and compositions of permeate, sap, and concentrate samples from the 2009 maple production season from operations that concentrated to varying levels with RO $(n=13)$. bdl = below the detection limit.

\begin{tabular}{|c|c|c|c|c|c|c|c|c|c|}
\hline & \multicolumn{3}{|c|}{ Permeate } & \multicolumn{3}{|c|}{ Sap } & \multicolumn{3}{|c|}{ Concentrate } \\
\hline & Mean & Min. & Max. & Mean & Min. & Max. & Mean & Min. & Max. \\
\hline${ }^{\circ}$ Brix & $0.1 \pm 0.01$ & 0.0 & 0.1 & $2.0 \pm 0.08$ & 1.5 & 2.4 & $14.3 \pm 0.95$ & 8.9 & 19.8 \\
\hline $\mathrm{pH}$ & $5.86 \pm 0.07$ & 5.41 & 6.36 & $6.61 \pm 0.09$ & 6.10 & 7.40 & $6.96 \pm 0.07$ & 6.50 & 7.42 \\
\hline Conductivity $(\mu \mathrm{S} / \mathrm{cm})$ & $15.1 \pm 1.8$ & 4.8 & 25.0 & $497.2 \pm 17.4$ & 388.8 & 594.8 & $1996.1 \pm 126.3$ & 1291.8 & 2609.5 \\
\hline Sucrose (\%) & & & & $1.72 \pm 0.07$ & 1.26 & 2.14 & $13.1 \pm 0.9$ & 8.2 & 18.8 \\
\hline Glucose (\%) & & & & $0.032 \pm 0.011$ & 0.004 & 0.148 & $0.21 \pm 0.07$ & 0.05 & 0.95 \\
\hline Fructose (\%) & & & & $0.029 \pm 0.009$ & 0.004 & 0.126 & $0.17 \pm 0.05$ & 0.05 & 0.71 \\
\hline $\mathrm{Ca}(\mathrm{ppm})$ & & & & $49.1 \pm 3.3$ & 24.4 & 67.6 & $388.6 \pm 39.3$ & 170.2 & 564.7 \\
\hline $\mathrm{P}(\mathrm{ppm})$ & & & & $1.2 \pm 0.2$ & 0.45 & 2.20 & $10.0 \pm 2.6$ & 3.4 & 38.0 \\
\hline $\mathrm{K}(\mathrm{ppm})$ & & & & $64.6 \pm 2.5$ & 54.0 & 80.2 & $464.8 \pm 46.1$ & 216.2 & 735.1 \\
\hline $\mathrm{Mg}(\mathrm{ppm})$ & & & & $5.17 \pm 0.30$ & 3.12 & 7.25 & $38.3 \pm 3.7$ & 20.9 & 56.9 \\
\hline $\mathrm{Na}(\mathrm{ppm})$ & & & & $1.30 \pm 0.95$ & 0.16 & 12.69 & bdl & & \\
\hline $\mathrm{Fe}(\mathrm{ppm})$ & & & & $0.19 \pm 0.17$ & 0.01 & 2.17 & $1.6 \pm 1.2$ & 0.2 & 16.0 \\
\hline $\mathrm{Mn}(\mathrm{ppm})$ & & & & $3.84 \pm 0.55$ & 0.78 & 7.92 & $31.1 \pm 4.4$ & 6.1 & 59.7 \\
\hline $\mathrm{B}(\mathrm{ppm})$ & & & & bdl & & & $0.4 \pm 0.1$ & 0.0 & 0.7 \\
\hline $\mathrm{Cu}(\mathrm{ppm})$ & & & & $0.16 \pm 0.08$ & 0.02 & 1.03 & $0.5 \pm 0.1$ & 0.3 & 1.2 \\
\hline $\mathrm{Zn}(\mathrm{ppm})$ & & & & $0.26 \pm 0.04$ & 0.15 & 0.63 & $2.0 \pm 0.2$ & 1.0 & 3.4 \\
\hline $\mathrm{S}(\mathrm{ppm})$ & & & & $1.03 \pm 0.21$ & 0.38 & 3.45 & $5.3 \pm 1.1$ & 0.7 & 13.2 \\
\hline
\end{tabular}

range of concentration levels of the samples analyzed. However, the overall means for sap and permeate samples provide a portrait of the average composition of sap and permeate samples from a variety of producers across a single production season.

The sugar concentration of sap ranged from 1.5 to $2.4{ }^{\circ}$ Brix, with an average of $2.0{ }^{\circ} \mathrm{Brix}$ (Table 1). $\mathrm{pH}$ was near neutral to slightly acidic, ranging from 6.10 to 7.40 , with an average of 6.61 across all producers and time of season. Sucrose was the predominant carbohydrate, with trace amounts of glucose and fructose also present (Table 1). The minerals found in the highest concentrations were $\mathrm{K}$ and $\mathrm{Ca}$, with mean concentrations of 64.6 and $49.1 \mathrm{ppm}$, respectively. The minimum, maximum, and standard error values for the concentrations of all minerals analyzed illustrate that there is relatively substantial variability in the mineral composition of sap (Table 1). All of these observations are consistent with the available published composition data for maple sap [1] [9] [10].

Permeate samples were more acidic than sap, with an average value of 5.86, and ranging from 5.41 to 6.36 (Table 1). This is consistent with the properties of 
membranes used in separation processes. Because membranes are permeable to carbon dioxide gas but not to the bicarbonate ions which typically balance the effects of $\mathrm{CO}_{2}$ on $\mathrm{pH}$, the $\mathrm{pH}$ of the concentrated material will typically be slightly higher, and that of the permeate water slightly lower, than the incoming material being concentrated. This expected pattern can likewise be observed in the concentrate samples, which had an average $\mathrm{pH}$ slightly higher than that of the sap samples (Table 1 ). The average conductivity of permeate samples was low, $15.1 \mu \mathrm{S} / \mathrm{cm}$, well below the generally recommended maximum of $50 \mu \mathrm{S} / \mathrm{cm}$ [12], indicating that the membranes used by the RO machines included in this study were generally not allowing a large quantity of mineral ions to pass through into permeate. Likewise, the average ${ }^{\circ}$ Brix of permeate samples was also low, $0.1 \%$, indicating that the membranes of RO units in this study were also not allowing significant amounts of sucrose to pass through and be lost in permeate.

\subsection{Producer-Level Means}

Table 2 presents the overall means for the sample sets from each producer, which can provide information not discernible from the overall means. Only 1 set of samples had conductivity values in permeate greater than the maximum recommended threshold (Producer A, Table 2). Mineral analysis of these samples confirmed that the conductivity was likely elevated by the presence of mineral ions, in particular $\mathrm{Ca}, \mathrm{K}, \mathrm{Na}$, and $\mathrm{Mn}$, indicating that the membranes of this RO unit were likely not functioning optimally, and were allowing elevated levels of mineral ions to pass through into the permeate. Because of this, the data from this producer's samples were not included in any other analysis (e.g. Table 1, Table 3, and Table 4).

The average $\mathrm{pH}$ of sample sets from all producers exhibited the expected pattern-the $\mathrm{pH}$ of concentrate was slightly higher, and permeate slightly lower, than that of the starting sap material (Table 2). There was no trend for the $\mathrm{pH}$ of concentrate with increasing concentration level. However, as would be expected, the concentration of all constituents increased with increasing concentration (Table 2). Reflecting that, the conductivity of concentrate samples increased with increasing concentration levels. There were no trends in the $\mathrm{pH}$ or conductivity of permeate samples with increasing concentration level (Table 2).

The carbohydrate and mineral compositions were analyzed for the permeate samples from 2 producers, $\mathrm{C}$ and L (Table 2). These data illustrate the presence of $\mathrm{Ca}, \mathrm{K}, \mathrm{Na}$, and $\mathrm{Mn}$, and highlight the fact that membranes will allow the passage of some mineral ions [12]. However, the retention rates for these minerals in these samples were still quite high, with averages of $99 \%, 96 \%, 61 \%$, and $99 \%$, respectively.

\subsection{Season Timepoint Means}

Table 3 shows the average composition of permeate, sap, and concentrate samples at early, middle, and late timepoints of the maple production season. The 
Table 2. Mean ( \pm standard error of the mean) physicochemical properties and compositions of sets of permeate (P), sap (S), and concentrate (C) samples collected at Early, Middle, and Late timepoints of the 2009 maple production season by each of 14 maple operations (A through $\mathrm{N}$ ) which used a range of RO concentration levels. bdl = below the detection limit.

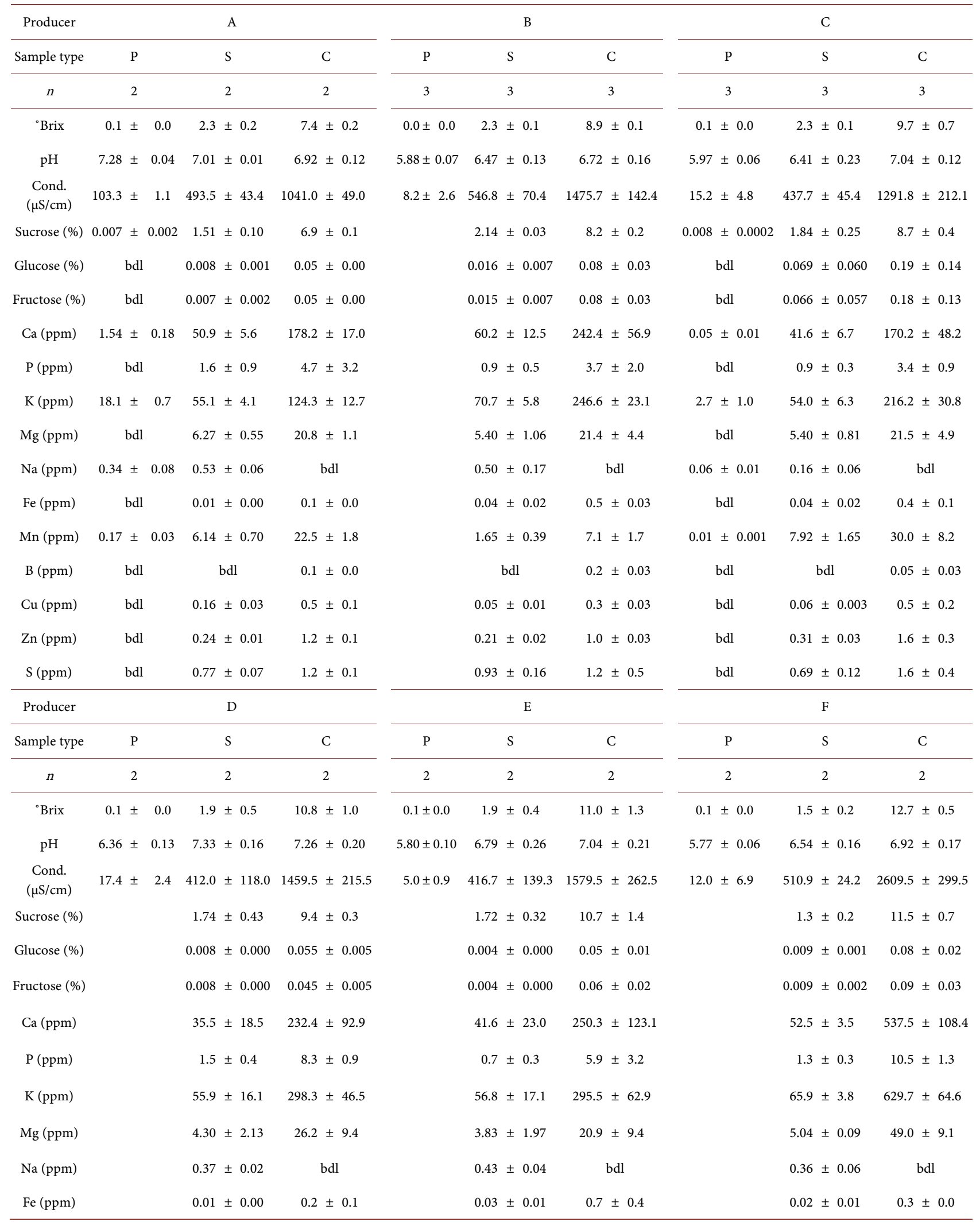




\section{Continued}

\begin{tabular}{|c|c|c|c|c|c|c|c|c|c|c|c|}
\hline Mn (ppm) & & & $0.78 \pm 0.43$ & $6.1 \pm 2.5$ & & 1.74 & \pm 0.97 & $11.3 \pm 5.2$ & & $3.52 \pm 0.36$ & $36.6 \pm 7.9$ \\
\hline $\mathrm{B}(\mathrm{ppm})$ & & & bdl & $0.4 \pm 0.1$ & & & bdl & $0.1 \pm 0.0$ & & bdl & $0.7 \pm 0.2$ \\
\hline $\mathrm{Zn}(\mathrm{ppm})$ & & & $0.21 \pm 0.01$ & $1.5 \pm 0.2$ & & 0.24 & \pm 0.03 & $1.5 \pm 0.2$ & & $0.18 \pm 0.02$ & $2.0 \pm 0.3$ \\
\hline $\mathrm{S}(\mathrm{ppm})$ & & & $0.61 \pm 0.28$ & $1.9 \pm 1.1$ & & 0.70 & \pm 0.29 & $0.7 \pm 0.0$ & & $1.15 \pm 0.40$ & $13.2 \pm 6.5$ \\
\hline Producer & & & G & & & & $\mathrm{H}$ & & & I & \\
\hline Sample type & $\mathrm{P}$ & & S & $\mathrm{C}$ & $\mathrm{P}$ & & $\mathrm{S}$ & $\mathrm{C}$ & $\mathrm{P}$ & $\mathrm{S}$ & $\mathrm{C}$ \\
\hline$n$ & 3 & & 3 & 3 & 3 & & 3 & 3 & 2 & 2 & 2 \\
\hline${ }^{\circ}$ Brix & $0.1 \pm$ & 0.0 & $2.4 \pm 0.1$ & $14.6 \pm 0.5$ & $0.1 \pm 0.0$ & 2.1 & \pm 0.1 & $15.2 \pm 0.6$ & $0.1 \pm 0.0$ & $2.1 \pm 0.5$ & $15.9 \pm 0.7$ \\
\hline $\begin{array}{l}\text { Cond. } \\
(\mu \mathrm{S} / \mathrm{cm})\end{array}$ & $4.8 \pm$ & 0.1 & $536.5 \pm 63.5$ & $1951.0 \pm 166.7$ & $17.3 \pm 7.6$ & 533.7 & \pm 33.3 & $2236.7 \pm 265.7$ & $16.4 \pm 1.3$ & $594.8 \pm 18.8$ & $2537.0 \pm 348.0$ \\
\hline Sucrose (\%) & & & $2.01 \pm 0.16$ & $13.4 \pm 0.7$ & & 1.83 & \pm 0.10 & $14.0 \pm 0.3$ & & $1.87 \pm 0.43$ & $14.7 \pm 1.2$ \\
\hline Glucose (\%) & & & $0.010 \pm 0.002$ & $0.10 \pm 0.01$ & & 0.014 & \pm 0.004 & $0.11 \pm 0.03$ & & $0.020 \pm 0.010$ & $0.15 \pm 0.07$ \\
\hline Fructose (\%) & & & $0.010 \pm 0.002$ & $0.09 \pm 0.02$ & & 0.010 & \pm 0.003 & $0.09 \pm 0.03$ & & $0.020 \pm 0.010$ & $0.12 \pm 0.05$ \\
\hline $\mathrm{Ca}(\mathrm{ppm})$ & & & $54.5 \pm 12.7$ & $355.9 \pm 58.8$ & & 67.6 & \pm 7.61 & $564.7 \pm 102.9$ & & $60.1 \pm 3.35$ & $539.2 \pm 138.0$ \\
\hline $\mathrm{P}(\mathrm{ppm})$ & & & $0.6 \pm 0.2$ & $3.8 \pm 1.7$ & & 0.8 & \pm 0.48 & $5.7 \pm 2.6$ & & $0.5 \pm 0.25$ & $4.2 \pm 0.5$ \\
\hline $\mathrm{K}(\mathrm{ppm})$ & & & $72.9 \pm 9.1$ & $425.0 \pm 36.5$ & & 59.6 & \pm 2.28 & $477.6 \pm 58.6$ & & $80.2 \pm 6.00$ & $672.4 \pm 91.5$ \\
\hline $\mathrm{Mg}(\mathrm{ppm})$ & & & $5.41 \pm 1.10$ & $34.8 \pm 4.8$ & & 7.25 & \pm 0.94 & $55.3 \pm 10.8$ & & $5.73 \pm 0.50$ & $52.0 \pm 14.1$ \\
\hline $\mathrm{Na}(\mathrm{ppm})$ & & & $0.33 \pm 0.10$ & bdl & & 0.26 & \pm 0.10 & bdl & & $0.32 \pm 0.03$ & bdl \\
\hline Mn (ppm) & & & $4.58 \pm 0.93$ & $31.2 \pm 4.8$ & & 5.54 & \pm 0.85 & $44.7 \pm 9.2$ & & $3.82 \pm 0.38$ & $33.9 \pm 9.0$ \\
\hline $\mathrm{B}(\mathrm{ppm})$ & & & bdl & $0.2 \pm 0.0$ & & & bdl & $0.7 \pm 0.2$ & & bdl & $0.6 \pm 0.1$ \\
\hline $\mathrm{Cu}(\mathrm{ppm})$ & & & $1.03 \pm 0.98$ & $0.3 \pm 0.03$ & & 0.05 & \pm 0.00 & $0.4 \pm 0.03$ & & $0.13 \pm 0.06$ & $0.6 \pm 0.1$ \\
\hline $\mathrm{Zn}(\mathrm{ppm})$ & & & $0.63 \pm 0.43$ & $1.6 \pm 0.03$ & & 0.27 & \pm 0.003 & $2.6 \pm 0.4$ & & $0.20 \pm 0.01$ & $1.9 \pm 0.3$ \\
\hline $\mathrm{S}(\mathrm{ppm})$ & & & $0.85 \pm 0.23$ & $5.0 \pm 0.6$ & & 1.02 & \pm 0.12 & $10.4 \pm 2.3$ & & $0.92 \pm 0.10$ & $10.5 \pm 3.2$ \\
\hline Producer & & & $\mathrm{J}$ & & & & $\mathrm{K}$ & & & $\mathrm{L}$ & \\
\hline Sample type & $\mathrm{P}$ & & S & $\mathrm{C}$ & $\mathrm{P}$ & & S & $\mathrm{C}$ & $\mathrm{P}$ & S & $\mathrm{C}$ \\
\hline$n$ & 1 & & 1 & 1 & 3 & & 3 & 3 & 3 & 3 & 3 \\
\hline${ }^{\circ}$ Brix & 0.1 & & 2.0 & 16.1 & $0.1 \pm 0.0$ & 2.1 & \pm 0.1 & $17.1 \pm 1.0$ & $0.1 \pm 0.0$ & $1.9 \pm 0.2$ & $17.3 \pm 0.9$ \\
\hline $\mathrm{pH}$ & 5.80 & & 7.21 & 7.06 & $5.41 \pm 0.21$ & 6.10 & \pm 0.15 & $6.50 \pm 0.16$ & $5.97 \pm 0.16$ & $6.41 \pm 0.21$ & $7.20 \pm 0.05$ \\
\hline $\begin{array}{l}\text { Cond. } \\
(\mu \mathrm{S} / \mathrm{cm})\end{array}$ & 25.0 & & 510.9 & 1741.0 & $16.5 \pm 5.3$ & 511.0 & \pm 55.4 & $1929.7 \pm 114.8$ & $21.2 \pm 2.1$ & $524.7 \pm 36.1$ & $2392.7 \pm 257.5$ \\
\hline Sucrose (\%) & & & 1.83 & 15.5 & & 1.46 & \pm 0.22 & $13.7 \pm 2.4$ & $0.029 \pm 0.011$ & $1.60 \pm 0.21$ & $15.8 \pm 1.1$ \\
\hline Glucose (\%) & & & 0.020 & 0.13 & & 0.148 & \pm 0.078 & $0.95 \pm 0.65$ & bdl & $0.025 \pm 0.011$ & $0.22 \pm 0.08$ \\
\hline Fructose (\%) & & & 0.020 & 0.11 & & 0.126 & \pm 0.063 & $0.71 \pm 0.60$ & bdl & $0.023 \pm 0.010$ & $0.19 \pm 0.06$ \\
\hline $\mathrm{Ca}(\mathrm{ppm})$ & & & 41.9 & 312.8 & & 46.1 & \pm 14.0 & $436.0 \pm 124.2$ & $1.03 \pm 0.25$ & $54.5 \pm 9.0$ & $544.6 \pm 88.8$ \\
\hline $\mathrm{P}(\mathrm{ppm})$ & & & 2.2 & 18.4 & & 2.1 & \pm 1.5 & $5.5 \pm 3.4$ & bdl & $0.7 \pm 0.2$ & $8.3 \pm 0.8$ \\
\hline $\mathrm{K}(\mathrm{ppm})$ & & & 77.9 & 443.9 & & 58.2 & \pm 3.3 & $472.1 \pm 39.7$ & $2.6 \pm 0.3$ & $64.7 \pm 3.5$ & $563.6 \pm 70.4$ \\
\hline
\end{tabular}




\section{Continued}

\begin{tabular}{|c|c|c|c|c|c|c|c|c|c|c|c|c|c|c|}
\hline $\mathrm{Mg}(\mathrm{ppm})$ & & & & 4.76 & 33.5 & & 4.76 & \pm 1.14 & $40.2 \pm 8.7$ & & bdl & & $6.27 \pm 0.94$ & $56.9 \pm 8.08$ \\
\hline $\mathrm{Na}(\mathrm{ppm})$ & & & & .46 & bdl & & 12.69 & \pm 12.20 & bdl & 0.14 & \pm & 0.01 & $0.41 \pm 0.12$ & bdl \\
\hline $\mathrm{Mn}(\mathrm{ppm})$ & & & & 2.94 & 24.5 & & 4.39 & \pm 1.39 & $40.4 \pm 11.8$ & 0.07 & \pm & 0.003 & $5.64 \pm 0.89$ & $59.7 \pm 10.6$ \\
\hline B (ppm) & & & & bdl & 0.4 & & & bdl & $0.6 \pm 0.1$ & & bdl & & bdl & $0.2 \pm 0.1$ \\
\hline $\mathrm{Cu}(\mathrm{ppm})$ & & & & .24 & 0.4 & & 0.38 & \pm 0.32 & $0.6 \pm 0.1$ & & bdl & & $0.04 \pm 0.003$ & $0.7 \pm 0.1$ \\
\hline $\mathrm{Zn}(\mathrm{ppm})$ & & & & .15 & 1.6 & & 0.35 & \pm 0.03 & $3.0 \pm 0.4$ & & bdl & & $0.22 \pm 0.02$ & $2.8 \pm 0.2$ \\
\hline $\mathrm{S}(\mathrm{ppm})$ & & & & 0.61 & 1.9 & & 3.45 & \pm 2.64 & $5.4 \pm 1.6$ & & bdl & & $1.06 \pm 0.32$ & $5.9 \pm 2.2$ \\
\hline Producer & & & & M & & & & $\mathrm{N}$ & & & & & & \\
\hline$n$ & 3 & & 3 & & 3 & 2 & & 2 & 2 & & & & & \\
\hline${ }^{\circ}$ Brix & $0.1 \pm 0.0$ & 2.1 & \pm & 0.1 & $17.5 \pm 0.3$ & $0.1 \pm 0.0$ & 1.5 & \pm 0.4 & $19.8 \pm 0.7$ & & & & & \\
\hline $\mathrm{pH}$ & $5.93 \pm 0.09$ & 6.49 & \pm & 0.17 & $6.81 \pm 0.15$ & $6.10 \pm 0.10$ & 7.09 & \pm 0.17 & $7.10 \pm 0.04$ & & & & & \\
\hline $\begin{array}{l}\text { Cond. } \\
(\mu \mathrm{S} / \mathrm{cm})\end{array}$ & $13.4 \pm 2.5$ & 539.4 & \pm & 83.4 & $2275.7 \pm 190.9$ & $23.3 \pm 10.7$ & 388.8 & \pm 93.1 & $2470.0 \pm 21.0$ & & & & & \\
\hline Sucrose (\%) & & 1.81 & \pm & 0.08 & $15.8 \pm 1.4$ & & 1.26 & \pm 0.29 & $18.8 \pm 1.4$ & & & & & \\
\hline Glucose (\%) & & 0.050 & \pm & 0.035 & $0.38 \pm 0.24$ & & 0.018 & \pm 0.012 & $0.21 \pm 0.01$ & & & & & \\
\hline Fructose (\%) & & 0.043 & \pm & 0.028 & $0.30 \pm 0.18$ & & 0.019 & \pm 0.011 & $0.20 \pm 0.01$ & & & & & \\
\hline $\mathrm{Ca}(\mathrm{ppm})$ & & 58.23 & \pm & 17.29 & $518.3 \pm 131.8$ & & 24.4 & \pm 10.85 & $347.0 \pm 118.0$ & & & & & \\
\hline $\mathrm{P}(\mathrm{ppm})$ & & 1.43 & \pm & 0.93 & $14.5 \pm 8.4$ & & 1.9 & \pm 0.30 & $38.0 \pm 14.2$ & & & & & \\
\hline $\mathrm{Mg}(\mathrm{ppm})$ & & 5.90 & \pm & 1.71 & $49.8 \pm 13.0$ & & 3.12 & \pm 1.29 & $36.8 \pm 9.5$ & & & & & \\
\hline $\mathrm{Na}(\mathrm{ppm})$ & & 0.39 & \pm & 0.06 & $\begin{array}{c}\text { bd } \\
1\end{array}$ & & 0.24 & \pm 0.11 & bdl & & & & & \\
\hline $\mathrm{Fe}(\mathrm{ppm})$ & & 0.02 & \pm & 0.01 & $0.6 \pm 0.1$ & & 0.01 & \pm 0.00 & $0.4 \pm 0.0$ & & & & & \\
\hline Mn (ppm) & & 5.08 & \pm & 1.96 & $45.1 \pm 15.0$ & & 2.31 & \pm 1.26 & $33.8 \pm 11.0$ & & & & & \\
\hline$B(p p m)$ & & & bdl & & $0.6 \pm 0.1$ & & & bdl & $0.3 \pm 0.0$ & & & & & \\
\hline $\mathrm{Cu}(\mathrm{ppm})$ & & 0.04 & \pm & 0.01 & $0.3 \pm 0.03$ & & 0.02 & \pm 0.00 & $1.2 \pm 0.2$ & & & & & \\
\hline $\mathrm{Zn}(\mathrm{ppm})$ & & 0.23 & \pm & 0.07 & $2.3 \pm 0.5$ & & 0.15 & \pm 0.03 & $3.4 \pm 1.1$ & & & & & \\
\hline $\mathrm{S}(\mathrm{ppm})$ & & 0.97 & \pm & 0.37 & $8.5 \pm 3.6$ & & 0.38 & \pm 0.12 & $2.9 \pm 2.1$ & & & & & \\
\hline
\end{tabular}

values for sap samples exhibit some patterns across the season, and the data are also presented graphically to illustrate these (Figure 1). Sap sugar content as measured by a refractometer remained relatively stable across the production season (1.9, 2.2, and $1.9^{\circ}$ Brix, respectively). However, the carbohydrate data illustrate more nuanced changes, with sucrose concentrations rising slightly at mid-season before decreasing in late-season samples, and glucose and fructose concentrations increasing in late-season samples (Table 3, Figure 1). Conductivity generally increased from early to late season, and, likewise, the concentration of most minerals ( $\mathrm{Ca}, \mathrm{K}, \mathrm{Mg}, \mathrm{Mn}$, and $\mathrm{S}$ ) also generally increased. In contrast, concentrations of both $\mathrm{P}$ and $\mathrm{Na}$ decreased over the season, and those of 
Table 3. Mean ( \pm standard error of the mean), minimum, and maximum physicochemical properties and compositions of permeate, sap, and concentrate samples at Early, Middle, and Late timepoints of the 2009 maple production season from producers that concentrated to varying levels with $\mathrm{RO}$. bdl = below the detection limit.

\begin{tabular}{|c|c|c|c|c|c|c|c|c|c|}
\hline & \multicolumn{9}{|c|}{ Early } \\
\hline & \multicolumn{3}{|c|}{ Permeate } & \multicolumn{3}{|c|}{ Sap $^{\dagger}$} & \multicolumn{3}{|c|}{ Concentrate } \\
\hline & Mean & Min. & Max. & Mean & Min. & Max. & Mean & Min. & Max. \\
\hline$n$ & \multicolumn{3}{|c|}{11} & \multicolumn{3}{|c|}{10} & \multicolumn{3}{|c|}{11} \\
\hline${ }^{\circ}$ Brix & $0.1 \pm 0.0$ & 0.0 & 0.1 & $1.9 \pm 0.1$ & 1.1 & 2.4 & $13.9 \pm 1.1$ & 8.8 & 19.1 \\
\hline $\mathrm{pH}$ & $5.78 \pm 0.16$ & 5.06 & 6.55 & $7.17 \pm 0.07$ & 6.88 & 7.57 & $6.95 \pm 0.16$ & 6.22 & 7.62 \\
\hline Conductivity $(\mu \mathrm{S} / \mathrm{cm})$ & $16.1 \pm 3.1$ & 4.1 & 34.0 & $394.8 \pm 27.0$ & 277.4 & 510.9 & $1658.1 \pm 133.5$ & 990.3 & 2449.0 \\
\hline Sucrose (\%) & & & & $1.80 \pm 0.13$ & 0.97 & 2.18 & $12.6 \pm 1.1$ & 8.6 & 17.4 \\
\hline Glucose (\%) & & & & $0.008 \pm 0.001$ & 0.004 & 0.020 & $0.28 \pm 0.20$ & 0.04 & 2.26 \\
\hline Fructose (\%) & & & & $0.008 \pm 0.001$ & 0.004 & 0.020 & $0.24 \pm 0.17$ & 0.04 & 1.91 \\
\hline $\mathrm{Ca}(\mathrm{ppm})$ & & & & $31.4 \pm 3.9$ & 13.5 & 52.4 & $239.1 \pm 30.8$ & 107.0 & 380.7 \\
\hline $\mathrm{P}(\mathrm{ppm})$ & & & & $1.6 \pm 0.2$ & 1.0 & 3.3 & $15.4 \pm 4.3$ & 4.3 & 52.2 \\
\hline $\mathrm{K}(\mathrm{ppm})$ & & & & $53.8 \pm 4.1$ & 39.7 & 77.9 & $385.1 \pm 49.9$ & 195.0 & 721.6 \\
\hline $\mathrm{Mg}(\mathrm{ppm})$ & & & & $3.52 \pm 0.40$ & 1.83 & 5.36 & $25.6 \pm 3.1$ & 11.5 & 43.0 \\
\hline $\mathrm{Na}(\mathrm{ppm})$ & & & & $0.44 \pm 0.06$ & 0.07 & 0.75 & & & \\
\hline $\mathrm{Fe}(\mathrm{ppm})$ & & & & $0.02 \pm 0.004$ & 0.01 & 0.040 & $2.9 \pm 2.5$ & 0.1 & 28.2 \\
\hline Mn (ppm) & & & & $2.50 \pm 0.52$ & 0.36 & 5.21 & $20.0 \pm 3.5$ & 3.6 & 40.7 \\
\hline B (ppm) & & & & & & & $0.3 \pm 0.1$ & 0.0 & 0.7 \\
\hline $\mathrm{Cu}(\mathrm{ppm})$ & & & & $0.06 \pm 0.02$ & 0.02 & 0.24 & $0.5 \pm 0.1$ & 0.2 & 1.4 \\
\hline $\mathrm{Zn}(\mathrm{ppm})$ & & & & $0.21 \pm 0.01$ & 0.15 & 0.28 & $2.1 \pm 0.3$ & 1.0 & 4.4 \\
\hline \multirow[t]{4}{*}{$\mathrm{S}(\mathrm{ppm})$} & & & & $0.51 \pm 0.05$ & 0.26 & 0.78 & $3.7 \pm 1.3$ & 0.7 & 15.6 \\
\hline & \multicolumn{9}{|c|}{ Middle } \\
\hline & \multicolumn{3}{|c|}{ Permeate } & \multicolumn{3}{|c|}{ Sap } & \multicolumn{3}{|c|}{ Concentrate } \\
\hline & Mean & Min. & Max. & Mean & Min. & Max. & Mean & Min. & Max. \\
\hline$n$ & \multicolumn{3}{|c|}{12} & \multicolumn{3}{|c|}{10} & \multicolumn{3}{|c|}{12} \\
\hline${ }^{\circ}$ Brix & $0.1 \pm 0.0$ & 0.0 & 0.1 & $2.2 \pm 0.1$ & 1.6 & 2.5 & $14.6 \pm 1.1$ & 8.5 & 20.5 \\
\hline $\mathrm{pH}$ & $5.93 \pm 0.06$ & 5.68 & 6.47 & $6.75 \pm 0.07$ & 6.45 & 7.28 & $7.08 \pm 0.06$ & 6.82 & 7.63 \\
\hline Conductivity $(\mu \mathrm{S} / \mathrm{cm})$ & $13.2 \pm 2.3$ & 5.0 & 32.4 & $523.1 \pm 17.1$ & 435.6 & 594.7 & $2007.4 \pm 120.3$ & 1184.0 & 2493.0 \\
\hline Sucrose (\%) & & & & $1.88 \pm 0.09$ & 1.48 & 2.29 & $13.6 \pm 1.1$ & 8.0 & 20.1 \\
\hline Glucose (\%) & & & & $0.016 \pm 0.004$ & 0.004 & 0.04 & $0.12 \pm 0.02$ & 0.05 & 0.23 \\
\hline Fructose (\%) & & & & $0.015 \pm 0.004$ & 0.004 & 0.04 & $0.11 \pm 0.02$ & 0.04 & 0.20 \\
\hline $\mathrm{Ca}(\mathrm{ppm})$ & & & & $54.8 \pm 4.0$ & 35.2 & 74.4 & $400.4 \pm 37.0$ & 138.8 & 631.7 \\
\hline $\mathrm{P}(\mathrm{ppm})$ & & & & $1.0 \pm 0.2$ & 0.4 & 2.2 & $6.7 \pm 1.7$ & 1.8 & 23.8 \\
\hline $\mathrm{K}(\mathrm{ppm})$ & & & & $69.8 \pm 2.9$ & 57.4 & 86.2 & $464.0 \pm 47.3$ & 176.7 & 748.6 \\
\hline $\mathrm{Mg}(\mathrm{ppm})$ & & & & $5.75 \pm 0.37$ & 4.18 & 8.21 & $38.7 \pm 3.5$ & 18.8 & 61.1 \\
\hline
\end{tabular}


Continued

\begin{tabular}{|c|c|c|c|c|c|c|c|c|c|}
\hline $\mathrm{Na}(\mathrm{ppm})$ & & & & $0.33 \pm 0.04$ & 0.13 & 0.57 & \multicolumn{3}{|c|}{ bdl } \\
\hline $\mathrm{Fe}(\mathrm{ppm})$ & & & & $0.02 \pm 0.003$ & 0.004 & 0.03 & $1.3 \pm 0.9$ & 0.2 & 11.2 \\
\hline $\mathrm{Mn}(\mathrm{ppm})$ & & & & $4.01 \pm 0.70$ & 1.21 & 7.66 & $30.7 \pm 4.6$ & 8.0 & 61.0 \\
\hline $\mathrm{B}(\mathrm{ppm})$ & & & & \multicolumn{2}{|c|}{ bdl } & & $0.3 \pm 0.07$ & 0.0 & 0.8 \\
\hline $\mathrm{Cu}(\mathrm{ppm})$ & & & & $0.04 \pm 0.01$ & 0.02 & 0.07 & $0.5 \pm 0.07$ & 0.2 & 1.0 \\
\hline $\mathrm{Zn}(\mathrm{ppm})$ & & & & $0.20 \pm 0.02$ & 0.12 & 0.28 & $1.8 \pm 0.2$ & 0.9 & 2.9 \\
\hline \multirow[t]{4}{*}{$\mathrm{S}(\mathrm{ppm})$} & & & & $0.84 \pm 0.07$ & 0.50 & 1.14 & $4.5 \pm 1.0$ & 0.7 & 12.5 \\
\hline & \multicolumn{9}{|c|}{ Late } \\
\hline & \multicolumn{3}{|c|}{ Permeate } & \multicolumn{3}{|c|}{ Sap } & \multicolumn{3}{|c|}{ Concentrate } \\
\hline & Mean & Min. & Max. & Mean & Min. & Max. & Mean & Min. & Max. \\
\hline$n$ & \multicolumn{3}{|c|}{9} & \multicolumn{3}{|c|}{8} & \multicolumn{3}{|c|}{9} \\
\hline${ }^{\circ}$ Brix & $0.1 \pm 0.01$ & 0.0 & 0.1 & $1.9 \pm 0.1$ & 1.3 & 2.4 & $14.3 \pm 1.0$ & 9.1 & 18.3 \\
\hline $\mathrm{pH}$ & $5.85 \pm 0.05$ & 5.69 & 6.14 & $6.33 \pm 0.10$ & 6.03 & 7.44 & $6.77 \pm 0.08$ & 6.45 & 7.38 \\
\hline Conductivity $(\mu \mathrm{S} / \mathrm{cm})$ & $14.2 \pm 2.3$ & 4.7 & 24.7 & $591.9 \pm 22.2$ & 502.9 & 704.5 & $2375.9 \pm 161.5$ & 1666.0 & 2909.0 \\
\hline Sucrose (\%) & & & & $1.53 \pm 0.11$ & 1.08 & 2.08 & $12.4 \pm 0.9$ & 8.1 & 15.9 \\
\hline Glucose (\%) & & & & $0.055 \pm 0.023$ & 0.010 & 0.190 & $0.31 \pm 0.08$ & 0.09 & 0.87 \\
\hline Fructose (\%) & & & & $0.050 \pm 0.021$ & 0.010 & 0.180 & $0.23 \pm 0.07$ & 0.03 & 0.65 \\
\hline $\mathrm{Ca}(\mathrm{ppm})$ & & & & $69.0 \pm 4.5$ & 52.0 & 92.7 & $576.4 \pm 61.0$ & 264.9 & 778.7 \\
\hline $\mathrm{P}(\mathrm{ppm})$ & & & & $0.5 \pm 0.2$ & 0.1 & 1.6 & $4.1 \pm 1.1$ & 0.6 & 11.8 \\
\hline $\mathrm{K}(\mathrm{ppm})$ & & & & $69.2 \pm 2.7$ & 60.3 & 80.0 & $534.0 \pm 56.6$ & 276.9 & 763.8 \\
\hline $\mathrm{Mg}(\mathrm{ppm})$ & & & & $7.09 \pm 0.46$ & 5.12 & 9.32 & $55.3 \pm 5.9$ & 28.3 & 75.6 \\
\hline $\mathrm{Na}(\mathrm{ppm})$ & & & & $0.24 \pm 0.04$ & 0.07 & 0.38 & \multicolumn{3}{|c|}{ bdl } \\
\hline $\mathrm{Fe}(\mathrm{ppm})$ & & & & $0.04 \pm 0.01$ & 0.02 & 0.08 & $1.4 \pm 0.9$ & 0.2 & 8.5 \\
\hline $\mathrm{Mn}(\mathrm{ppm})$ & & & & $6.02 \pm 1.00$ & 2.14 & 10.90 & $50.4 \pm 6.9$ & 9.6 & 77.4 \\
\hline B (ppm) & & & & \multicolumn{3}{|c|}{ bdl } & $0.5 \pm 0.1$ & 0.1 & 0.9 \\
\hline $\mathrm{Cu}(\mathrm{ppm})$ & & & & $0.06 \pm 0.02$ & 0.02 & 0.18 & $0.4 \pm 0.1$ & 0.2 & 0.8 \\
\hline $\mathrm{Zn}(\mathrm{ppm})$ & & & & $0.26 \pm 0.03$ & 0.19 & 0.37 & $2.4 \pm 0.3$ & 1.0 & 3.2 \\
\hline $\mathrm{S}(\mathrm{ppm})$ & & & & $1.27 \pm 0.11$ & 0.90 & 1.70 & $8.8 \pm 2.0$ & 0.7 & 19.6 \\
\hline
\end{tabular}

${ }^{\dagger}$ Four sap samples with outlier values for trace metals $(\mathrm{Fe}, \mathrm{Cu}, \mathrm{Zn})$ were removed from mean calculations to prevent obscuring seasonal patterns.

trace minerals $\mathrm{Fe}, \mathrm{Cu}$, and $\mathrm{Zn}$ showed no discernible seasonal pattern (Table 3, Figure 1). The increase in $\mathrm{Mn}$ over the course of the season is interesting to note, as Mn has been putatively associated with early-season membrane fouling observed by some producers, particularly at higher concentration levels (Carl Lapierre, personal communication). However, that Mn concentrations in sap were actually lowest at the beginning of the season suggests that the relationship between $\mathrm{Mn}$ and this early-season membrane fouling may be indirect or noncausal. The properties of permeate samples were similar across all timepoints 
Table 4. Mean ( \pm standard error of the mean), minimum, and maximum differences between actual concentration of constituents in concentrate samples and the predicted concentration of each based on the concentration factor of sap to concentrate of each sample (predicted minus actual, $n=13$ ). Fixed effect parameter estimates and $p$-values are for repeated measures analysis of variance for each parameter of concentration factor and the difference between predicted and actual concentrations (with producer as the repeated measure, $n=32$ ).

\begin{tabular}{cccccc}
\hline & \multicolumn{5}{c}{ Differences-Predicted minus actual } \\
\hline & Mean & Min & Max & Estimate & $p$ \\
\hline Glucose (\%) & $0.03 \pm 0.02$ & -0.03 & 0.2 & 0.003 & 0.8339 \\
Fructose (\%) & $0.04 \pm 0.03$ & -0.04 & 0.3 & 0.009 & 0.5979 \\
$\mathrm{Ca}(\mathrm{ppm})$ & $-31.6 \pm 7.9$ & -72.6 & 24.5 & -4.2 & 0.3434 \\
$\mathrm{P}(\mathrm{ppm})$ & $-0.4 \pm 1.5$ & -12.2 & 13.8 & -0.1 & 0.8826 \\
$\mathrm{~K}(\mathrm{ppm})$ & $15.4 \pm 16.2$ & -51.2 & 183.2 & -2.8 & 0.7269 \\
$\mathrm{Mg}(\mathrm{ppm})$ & $-0.5 \pm 0.8$ & -5.0 & 4.8 & 0.3 & 0.4939 \\
$\mathrm{Fe}(\mathrm{ppm})$ & $-0.02 \pm 0.2$ & -0.5 & 2.6 & -0.01 & 0.8582 \\
$\mathrm{Mn}(\mathrm{ppm})$ & $-2.7 \pm 0.8$ & -5.9 & 5.2 & -0.7 & 0.0242 \\
$\mathrm{Cu}(\mathrm{ppm})$ & $0.7 \pm 0.5$ & -0.9 & 5.6 & -0.04 & 0.8951 \\
$\mathrm{Zn}(\mathrm{ppm})$ & $-0.2 \pm 0.2$ & -1.2 & 2.1 & -0.1 & 0.3463 \\
$\mathrm{~S}(\mathrm{ppm})$ & $2.7 \pm 2.1$ & -2.9 & 26.2 & 1.0 & 0.3753 \\
\hline
\end{tabular}

Sucrose was not included in this analysis, as it is confounded with concentration factor; B and Na were also excluded, as the elements were below the detection limit in sap and concentrate samples, respectively.
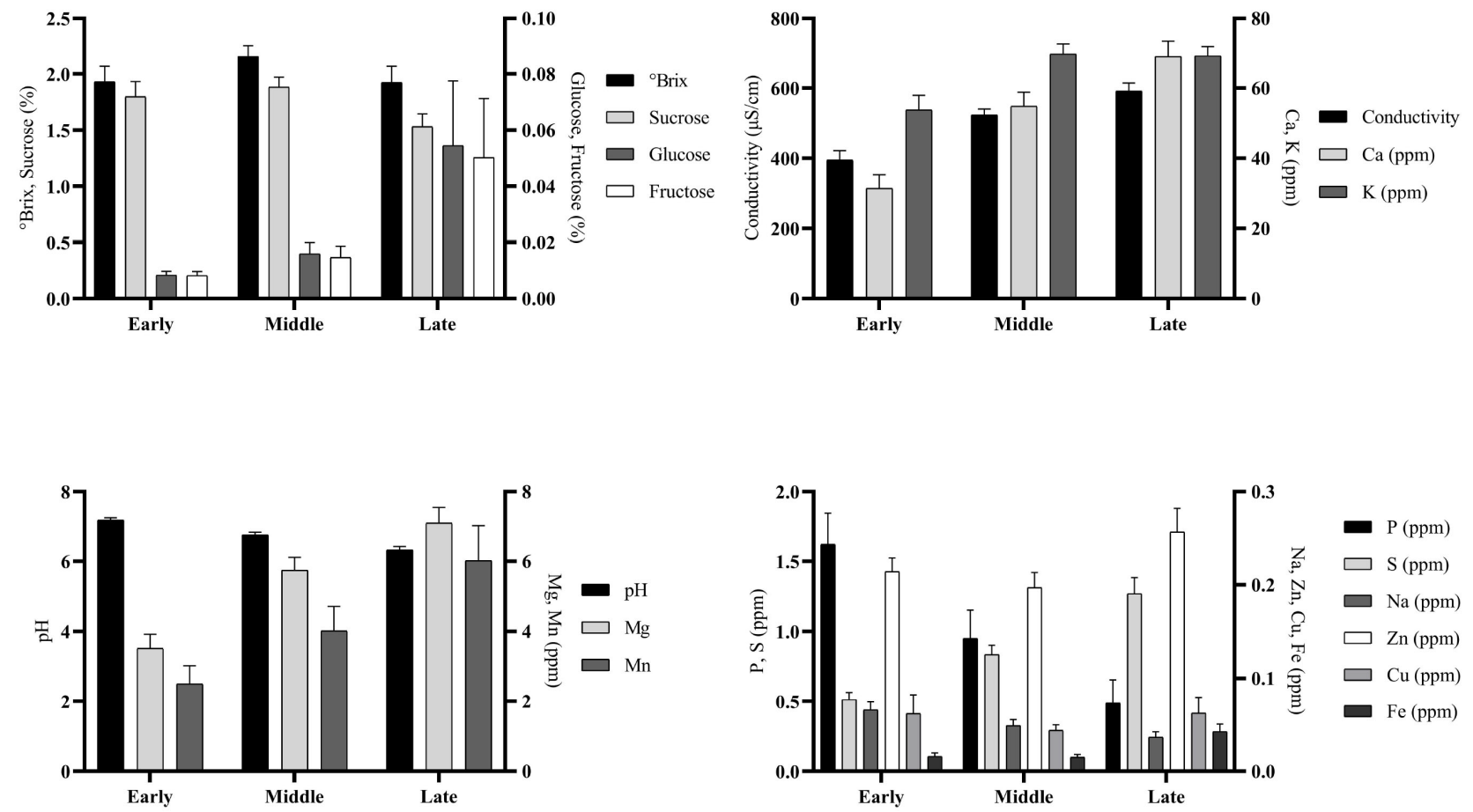

Figure 1. Mean physicochemical properties and compositions of maple sap samples at Early, Middle, and Late timepoints of the 2009 maple production season. $n=10$ (Early), 10 (Middle), and 8 (Late). Error bars represent standard error of the mean. 
(Table 3). Seasonal patterns cannot be examined for concentrate samples because the values are comprised of samples from such a wide range of concentrations.

\subsection{Differences in Predicted and Actual Concentration of Constituents in Concentrate}

Concern is sometimes expressed that $\mathrm{RO}$ could potentially allow the loss or disproportionate concentration of sap constituents, altering the fundamental composition of maple sap, and thereby resulting in unacceptable effects on the composition of syrup. However, if RO units and membranes are operating optimally, they should essentially simply concentrate sap, removing water without significant loss or addition of constituents present in the sap. If this is the case, the composition of constituents in concentrate should be very similar to what is predicted by the concentration factor between the initial sap material and the concentrate. The data in this study can be used to assess this, by comparing the actual concentration of constituents in the concentrate to that predicted by the concentration factor. Table 4 shows the average differences between the actual composition values of constituents in concentrate samples and the predicted concentration of each constituent based on the sap to concentrate concentration factor. These data illustrate that the differences between actual and predicted values are typically quite small, even for constituents known to pass through membranes in small amounts, such as $\mathrm{K}$ and $\mathrm{Ca}$ (Table 4). They also illustrate a substantial amount of variation, with all constituents being present in both higher and lower concentrations than predicted by the concentration factor in some samples, as illustrated by the minimum and maximum difference values (Table 4). Of particular interest are the results with respect to "invert sugar", glucose and fructose. It is often presumed that concentrate will have a proportionally higher invert concentration than the raw sap from which it was generated due to increased microbial activity with greater sugar concentrations. However, the concentrate samples in this study contained similar quantities of glucose and fructose to those that were predicted by the concentration factor, with average differences reflecting lower concentrations in concentrate than predicted by the concentration factor (Table 4). This is consistent with results observed in a previous analysis of sap and concentrate composition from a single RO [8], and suggests invert levels are not typically disproportionately elevated in concentrate. This analysis does not preclude the existence of effects on other constituents not analyzed, such as phenolics or amino acids; however the size and properties of most other sap constituents would make significant loss or disproportionate concentration unlikely. And although variability was observed, in general this analysis suggests that reverse osmosis in maple production functions generally as simple concentration, not altering the fundamental proportional composition of maple sap.

An additional analysis was used to determine if the level of concentration used 
was related to reverse osmosis performance. Statistical analyses were conducted to determine if any significant relationships existed between the concentration factor and the difference between the predicted and actual concentration of any constituents in the concentrate. For most constituents, there was no significant relationship between the concentration factor and the differences between the predicted and actual concentration of the concentrate (Table 4). However, the differences between the predicted and actual concentrations of Mn were significantly related to the concentration factor, with more negative values at higher concentration levels (Table 4). This indicates that at higher concentration levels, there tended to be more $\mathrm{Mn}$ in concentrate than predicted by the concentration factor. This could suggest that the interaction of Mn (or a Mn-containing sap constituent) and RO membranes might be altered in some way at higher sap concentration levels, leading to larger quantities than predicted by the concentration factor to be retained in the concentrate. If true, this could have important implications for RO operation at higher sap concentration levels, and the mechanism through which this occurs would be critical to elucidate. However, although this analysis is suggestive, it is possible that the relationship is confounded by some factor not accounted for, or by inherent errors or biases in the analyses. The behavior of $\mathrm{Mn}$ and interaction with $\mathrm{RO}$ membranes at higher concentration levels should be further investigated.

\section{Conclusion}

In summary, the mean overall, producer-level, and seasonal timepoint compositions and physiochemical properties provide a snapshot of sap, concentrate, and permeate samples from a variety of concentration levels. The data illustrate that $\mathrm{RO}$ is fundamentally a concentration process, and as such is unlikely to significantly alter properties of resulting syrup, consistent with results observed in pervious experiments [2]-[8].

\section{Acknowledgements}

Funding support for this project was provided by United States Department of Agriculture Cooperative State Research, Education, and Extension Service (USDA CSREES) grant 2008-34157-19186. We thank the participating maple producers and Alan Howard for their assistance in completing this research.

\section{Conflicts of Interest}

The authors declare no conflicts of interest regarding the publication of this paper.

\section{References}

[1] Perkins, T.D. and van den Berg, A.K. (2009) Maple Syrup-Production, Composition, Chemistry, and Sensory Characteristics. In: Taylor, S.L., Ed., Advances in Food and Nutrition Research, Academic Press, San Diego, CA, 103-144. 
[2] Allard, G. (1981) Comparaison des sirops d'érable produits par osmose et par évaporation conventionnelle sur la base de leurs propriétés physique, chimiques et organoleptiques. Journée d'information sur la technique de l'osmose inverse. Centre Acer publication No: 101-HPG-0981.

http://www.centreacer.qc.ca/uploaded/Publications/62_Fr.pdf

[3] Morselli, M.F., Whalen, M.L., Baggett, K.L. and Sendak, P.E. (1982) Quality of Syrup Produced from Sap Concentrated by Reverse Osmosis (RO). Maple Syrup Journal, 6, 18.

[4] Sendak, P.E. and Morselli, M.F. (1984) Reverse Osmosis in the Production of Maple syrup. Forest Products Journal, 34, 57-61.

[5] van den Berg, A.K., Perkins, T.D., Isselhardt, M.L., Godshall, M.A. and Lloyd, S.W. (2011) Effects of Producing Maple Syrup from Concentrated and Reconstituted Sap of Different Sugar Concentrations. International Sugar Journal, 113, 35-44.

[6] van den Berg, A.K., Perkins, T.D., Isselhardt, M.L., Godshall, M.A. and Lloyd, S.W. (2012) Maple Syrup Production with Sap Concentrated to High Levels by Membrane Separation: Effects on Syrup Chemical Composition and Flavor. International Sugar Journal, 114, 572-576.

[7] van den Berg, A.K., Perkins, T.D., Isselhardt, M.L., Godshall, M.A. and Lloyd, S.W. (2014) Effects of Membrane Separation on Maple Syrup Composition and Flavor. International Sugar Journal, 116, 656-665.

[8] van den Berg, A.K., Perkins, T.D., Isselhardt, M.L., Godshall, M.A. and Lloyd, S.W. (2015) Effects of Sap Concentration with Reverse Osmosis on Syrup Composition and Flavor-A Summary of Experiments Conducted at the University of Vermont Proctor Maple Research Center. Maple Digest, 54, 11-33.

[9] Stuckel, J.G. and Low, N.H. (1996) The Chemical Composition of 80 Pure Maple Syrup Samples Produced in North America. Food Research International, 29, 373-379. https://doi.org/10.1016/0963-9969(96)00000-2

[10] Perkins, T.D., Morselli, M.F., van den Berg, A.K. and Wilmot, T.R. (2006) Maple Chemistry and Quality. In: Heiligmann, R.B., Koelling, M.R. and Perkins, T.D., Eds., North American Maple Syrup Producers Manual, 2nd Edition, The Ohio State University, Ohio, 294-300.

[11] Hornung, R.W. and Reed, L. (1990) Estimation of Average Concentration in the Presence of Nondetectable Values. Applied Occupational and Environmental Hygiene, 5, 46-51. https://doi.org/10.1080/1047322X.1990.10389587

[12] Allard, G.B. and Belizle, M. (2004) Cahier de Transfert Technologique en Acériculture. Section 6: Concentration partielle de l'eau érable par osmose inversée. Centre de référence en agriculture et agroalimentaire du Québec, La Pocatière, 38 p. 